\title{
Loop-mediated isothermal amplification of a single DNA molecule in polyacrylamide gel-based microchamber
}

\author{
Liza Lam • Shouichi Sakakihara $\cdot$ Koji Ishizuka • \\ Shoji Takeuchi • Hideyuki F. Arata • Hiroyuki Fujita • \\ Hiroyuki Noji
}

Published online: 26 March 2009

(C) Springer Science + Business Media, LLC 2009

\section{Erratum to: Biomed Microdevices (2008) 10:539-546 \\ DOI 10.1007/s10544-008-9163-x}

The original version of this article unfortunately contained a mistake. An incorrect set of primers was entered into the manuscript. In section 2.1, 2nd sentence onwards till end of section 2.1, the correct set of primers used in the experiment should read:

Using the LAMP primer designing software (Primer Explorer, Japan), the following primers were generated FIP contained F1c (21 nt), a tttt linker and the complementary sequence of F2c (20 nt): 5'-gcagaacgggcattccetgtt-ttttcggatactcgcaccgaaaat-3'; BIP contained the complementary sequence of B1 (20 nt), a tttt linker and B2 (18 nt): 5'-cattaccettgccaccgect-tttt-ttcagcatccetttcggc-3'; F3: 5'tcaacaaaaagcagctggct-3'; and B3: 5'-gcgcagctttcgttctca-3'.

In the Appendix section, an additional ' $\mathrm{g}$ ' was missing from the start of the sequence due to a typo-graphical error. Therefore, it should read as:

Sequence of $2 \mathrm{kbp}$ target

5'-gggcggegacctcgegggttttcgctatttatgaaaattttccggtt...

The online version of the original article can be found at http://dx.doi. org/10.1007/s10544-008-9163-x.

L. Lam $(\bowtie) \cdot$ S. Sakakihara $\cdot$ K. Ishizuka $\cdot$ H. Noji $(\bowtie)$

The Institute of Scientific and Industrial Research,

Osaka University,

Osaka, Japan

e-mail: lizalam@sanken.osaka-u.ac.jp

e-mail: hnoji@sanken.osaka-u.ac.jp

S. Takeuchi $\cdot$ H. F. Arata $\cdot$ H. Fujita

Center for International Research on MicroMechatronics,

Institute of Industrial Science, University of Tokyo,

Tokyo, Japan 